\title{
Bright and dark solitary waves in a one-dimensional spin-polarized gas of fermionic atoms with p-wave interactions in a hard-wall trap
}

\author{
M. D. Girardeau ${ }^{1, \text { 田 }}$ and E. M. Wright ${ }^{1,2, \text { 团 }}$ \\ ${ }^{1}$ College of Optical Sciences, University of Arizona, Tucson, AZ 85721, USA \\ ${ }^{2}$ Department of Physics, University of Arizona, Tucson, AZ 85721, USA
}

(Dated: October 31, 2018)

\begin{abstract}
In this paper we elucidate the physics underlying the fact that both bright and dark solitary waves can arise in a one-dimensional spin-polarized gas of fermionic atoms with attractive threedimensional p-wave interactions in a hard-wall trap. This is possible since the one-dimensional fermion system can be mapped to a system of bosons described by the Lieb-Linger model with either repulsive or attractive delta-function interactions which can support solitary waves.
\end{abstract}

PACS numbers: 03.75.-b,05.30.Fk

Advances in experimental techniques for probing ultracold gases have resulted in a shift in emphasis in theoretical and experimental work in recent years from effective field approaches to more refined methods capable of dealing with strong correlations. Such strong correlations occur in ultracold gases confined in de Broglie waveguides with transverse trapping so tight that the atomic dynamics is essentially one-dimensional (1D) 1], with confinement-induced resonances [1, 2] allowing Feshbach resonance tuning [3] of the effective 1D interactions to very large values. This has led to experimental verification [4, 5, 6] of the fermionization of bosonic ultracold vapors in such geometries predicted by the Fermi-Bose (FB) mapping method [7], an exact mapping of a 1D gas of bosons with point hard core repulsions, the "TonksGirardeau" (TG) gas, to an ideal spin-aligned Fermi gas. The "fermionic Tonks-Girardeau" (FTG) gas [8, 9], a 1D spin-aligned Fermi gas with very strong attractive interactions, can be realized by a 3D p-wave Feshbach resonance as, e.g., in ultracold ${ }^{40} \mathrm{~K}$ vapor 10. It has been pointed out [2, 8, 9] that the generalized FB mapping [2, 8, 9, 11] can be exploited to map the ideal FTG gas with infinitely strong attractive interactions to the ideal Bose gas, leading to "bosonization" of many properties of this Fermi system. In a recent paper [12] we examined how the properties of such an FTG gas on a mesoscopic ring are changed for an even number $N$ of fermions when the strength of the atom-atom attraction is made finite, in which case there exists an FB mapping between the FTG gas and a system of $N$ bosons with repulsive deltafunction interactions.

It has been realized in recent years that the FB mapping method used to exactly solve the TG gas [7] and FTG gas [8, 9] is not restricted to the TG case of point hard core boson-boson repulsion and the ideal FTG case of infinite zero-range fermion-fermion attraction. In fact, the same unit antisymmetric mapping function used there, $A\left(x_{1}, \cdots, x_{N}\right)=\prod_{1 \leq j<\ell<N} \operatorname{sgn}\left(x_{j}-x_{\ell}\right)$, provides an exact mapping between bosons with delta-function repulsions $g_{1 D}^{B} \delta\left(x_{j}-x_{\ell}\right)$ of any strength, i.e., the Lieb-
Liniger (LL) model 13], and spin-aligned fermions with attractive interactions of a generalized FTG form with reciprocal fermionic coupling constant $g_{1 D}^{F}$; here $\operatorname{sgn}(x)$ is $+1(-1)$ if $x>0(x<0)$. All previous work, including [12] and a recent Bethe ansatz treatment of such a system in a hard-wall trap [14], have considered only the case where the strongly attractive spin-aligned Fermi gas maps to the LL model with repulsive interactions, corresponding to a negative $1 \mathrm{D}$ scattering length $a_{1 D}$. This restriction is not necessary and a minor change in the parameters results in a mapping of the FTG gas to an LL model with attractive interactions, corresponding to a positive scattering length $0<a_{1 D}<\infty$, an equally physical regime. The ideal FTG gas $\left|a_{1 D}\right| \rightarrow \infty$ lies on the "knife edge" between the attractive and repulsive LL regimes, where $a_{1 D}$ jumps discontinuously from $-\infty$ to $+\infty$, as might have been expected from the fact that it corresponds to a zero-energy scattering resonance. The purpose of the present paper is to elucidate how this works, and to compare and contrast the ground states of the spin-aligned Fermi gas in these two regimes. In particular, for the case of a hard-wall trap and using the mapping between the FTG and LL model, we expose that both bright and dark solitary waves can arise. The possibility of solitons in degenerate Fermi gases [15, 16, 17] has certainly been investigated previously, and the novelty in the present work is that it includes the p-wave fermionfermion interactions, highlights the relation between the Fermi and Bose systems, and exposes the role played by the solitary waves of the underlying Bose system.

$3 D$-wave resonance and induced $1 D$ interaction: $3 \mathrm{D}$ $\mathrm{p}$-wave Feshbach resonances have been observed in some species of ultracold gases, e.g., in ${ }^{40} \mathrm{~K}[10]$. When such a gas is contained in a de Broglie waveguide with tight transverse trapping so as to reach the regime of effectively $1 \mathrm{D}$ dynamics, such a $3 \mathrm{D}$ resonance leads to a nearby confinement-induced resonance (CIR) in the 1D scattering, and it has been shown [2, 8, , 9] that the 3D p-wave scattering length $a_{p}$ and $1 \mathrm{D}$ scattering length $a_{1 D}$ in the 
neighborhood of such a resonance have the connection

$$
a_{1 D}=\frac{6 V_{p}}{a_{\perp}^{2}}\left[1+12\left(V_{p} / a_{\perp}^{3}\right)|\zeta(-1 / 2,1)|\right]^{-1},
$$

where $a_{\perp}=\sqrt{\hbar / m_{\text {red }} \omega_{\perp}}$ is the transverse oscillator length, $V_{p}=a_{p}^{3}=-\lim _{k \rightarrow 0} \tan \delta_{p}(k) / k^{3}$ is the p-wave "scattering volume", $a_{p}$ is the p-wave scattering length, $\zeta(-1 / 2,1)=-\zeta(3 / 2) / 4 \pi=-0.2079 \ldots$ is the Hurwitz zeta function evaluated at $(-1 / 2,1)$, and $m_{\text {red }}=m / 2$ is the reduced mass. The expression (11) has a resonance at a negative critical value $V_{p}^{\text {crit }} / a_{\perp}^{3}=-0.4009 \cdots$, implying that the CIR only occurs when $a_{p}<0$. However, by varying an external magnetic field so as to sweep $a_{p}$ through the critical value, one can in principle vary $a_{1 D}$ from $-\infty$ to $+\infty$. The ideal FTG gas, which maps to the ideal Bose gas, correspond to the point where $a_{1 D}$ jumps discontinuously from $-\infty$ to $+\infty$, and nearby values of $a_{p}$ correspond to an "imperfect FTG gas", which we shall call herein simply an FTG gas. $a_{1 D}$ is defined implicitly by the contact condition [2, 8, 9]

$$
\Psi_{F}\left(x_{12}=0+\right)=-\Psi_{F}\left(x_{12}=0-\right)=-a_{1 D} \Psi_{F}^{\prime}\left(x_{12}=0 \pm\right)
$$

where $\Psi_{F}\left(x_{12}\right)$ is the fermionic relative wave function and the prime denotes differentiation.

Several different 1D pseudopotentials have been used in the literature to represent a 1D potential leading to this contact condition, but most of these involve combinations of delta functions and derivatives which make them physically opaque. However, the physics is clearly exhibited if one instead uses a suitable zero-range limit of a deep and narrow square well of depth $V_{0}$ and width $2 x_{0}$, with the zero-range limit $V_{0} \rightarrow \infty$ and $x_{0} \rightarrow 0$ carried out at constant $V_{0} x_{0}^{2}[8,9,12]$. Consider first the two-body problem $N=2$ in infinite space. The relative wave function $\Psi_{F}\left(x_{12}\right)$ of the ground state inside the well is $\sin \left(\kappa x_{12}\right)$ where the value of the positive parameter $\kappa$ is determined by the total energy (potential plus kinetic), or equivalently, by $a_{1 D}$. One finds $\kappa x_{0}=\frac{\pi}{2}+\frac{2 x_{0}}{\pi a_{1 D}}$ as $x_{0} \rightarrow 0$ [8, 9]. The ideal FTG gas (total energy zero, i.e., zeroenergy resonance) corresponds to infinite $a_{1 D}$ and hence $\kappa x_{0}=\frac{\pi}{2}$. The imperfect FTG gas studied in [12] has negative $a_{1 D}$ and positive total energy, i.e., inside the well the positive kinetic energy exceeds the negative potential energy $-V_{0}$ by a finite amount. What we wish to point out here is that exactly the same relation $\kappa x_{0}=\frac{\pi}{2}+\frac{2 x_{0}}{\pi a_{1 D}}$ also applies to the case where $a_{1 D}$ is positive and the negative potential energy exceeds the positive kinetic energy inside the well, resulting in a finite and negative total energy, i.e., a bound ground state. The generalized FB mapping [2, 8, 9, 11] $\Psi_{B}\left(x_{12}\right)=\operatorname{sgn}\left(x_{12}\right) \Psi_{F}\left(x_{12}\right)$ maps the fermionic wave function to a bosonic one $\Psi_{B}$ with the same scattering length $a_{1 D}$. The case $a_{1 D}<0$ of this mapping was illustrated in Fig. 1 of [12], and the case $a_{1 D}>0$ which we wish to study here is illustrated by the present Fig 1. For consistency of the mapping one should

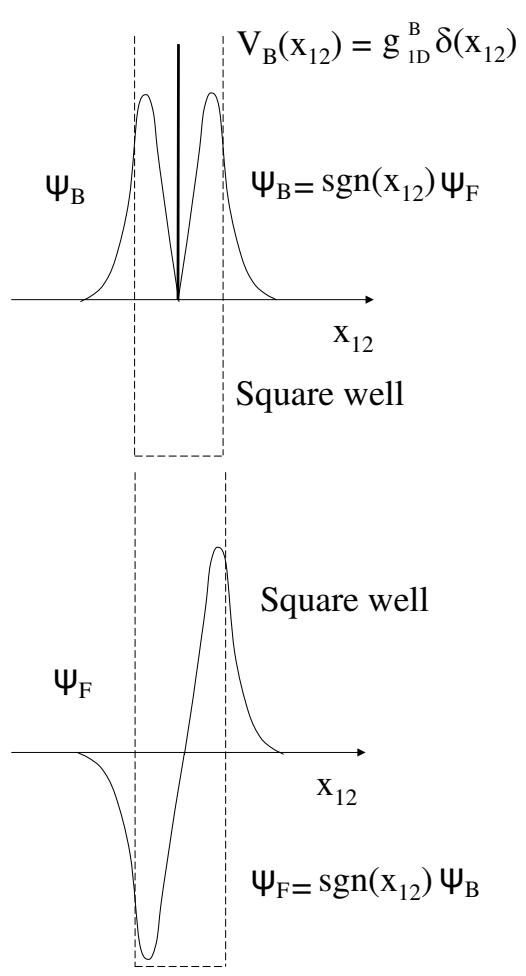

FIG. 1: Two-particle fermionic relative wave function $\Psi_{F}$ and mapped bosonic wave function $\Psi_{B}$ as a function of $x_{12}=x_{1}-x_{2}$, for the case where the negative potential energy slightly exceeds the positive kinetic energy, so the strongly attractive Fermi system maps to a Lieb-Liniger Bose gas with weak attractions. The potential for both $\Psi_{F}$ and $\Psi_{B}$ is a deep and narrow square well plus a point hard core, but in the zero-range limit its effect on $\Psi_{B}$ outside the well is the same as that of $V_{B}=g_{1 D}^{B} \delta\left(x_{12}\right)$, where here $g_{1 D}^{B}<0$.

add a zero-diameter hard core at $x_{12}=0$ to the potential, which has no effect on $\Psi_{F}[12]$. In the zero-range limit the effect of this potential on $\Psi_{B}$ outside the well is exactly the same as that of an LL delta function interaction $V_{B}=g_{1 D}^{B} \delta\left(x_{12}\right)$ with $g_{1 D}^{B}=-2 \hbar^{2} / m a_{1 D}<0$. The corresponding fermionic coupling constant is reciprocally related to $g_{1 D}^{B}$, i.e., $g_{1 D}^{F}=-2 \hbar^{2} a_{1 D} / m<0$ and $g_{1 D}^{B} g_{1 D}^{F}=\frac{4 \hbar^{4}}{m^{2}}$ [18]. Outside the well the fermionic and mapped bosonic wave functions are both decaying exponentials, i.e., the ground state is bound. In the case of enclosure in a longitudinal box or well, or trapping on a ring, the wave functions are modified at large distances in accordance with the boundary conditions and/or trap potential, but the above represents the exact behavior of the wave functions as $x_{12} \rightarrow 0$.

Many-fermion ground state: For a system of $N$ fermions the Fermi wave function may be related to the underlying Bose wave function via the mapping $\Psi_{F}=$ $A \Psi_{B}$, where $\Psi_{B}$ is the ground state of the N-boson LL Hamiltonian. The relative strengths of the induced 1D fermion interactions and the LL model interactions 
are quantified in the dimensionless coupling coefficients $\gamma_{F}=\frac{m g_{1 D}^{F} n}{\hbar^{2}}$ and $\gamma_{B}=\frac{m g_{1 D}^{B}}{\hbar^{2} n}$, respectively, where $n=\frac{N}{L}$ is the linear atomic density, and $\gamma_{F} \gamma_{B}=4$. The ideal FTG gas is realized in the limit $a_{1 D} \rightarrow-\infty$ so that $\gamma_{F} \rightarrow \infty$ and $\gamma_{B} \rightarrow 0$. Here we are interested in the limit of finite but very large $\left|a_{1 D}\right|$, implying $\left|\gamma_{F}\right|>>1$ and $\left|\gamma_{B}\right|<<1$. This is precisely the limit where the ground state properties of the LL Hamiltonian can be accurately captured using mean field theory in which all $\mathrm{N}$ bosons are assumed to occupy the same normalized single particle orbital $\phi$ that is determined as the ground state solution of the Gross-Pitaevskii equation (GPE) [19]

$$
\mu \phi=-\frac{\hbar^{2}}{2 m} \frac{d^{2} \phi}{d x^{2}}+g_{1 D}^{B}(N-1)|\phi|^{2} \phi,
$$

with $\mu$ the chemical potential, and the GPE is to be solved subject to the hard-wall boundary conditions $\phi(0)=\phi(L)=0$ for a trap of length L. (We employ a hard-wall trap due to the fact that the solutions of Eq. (3) are well known, but our general findings would also apply to a harmonic trap). The $N$-particle fermion wave function can then be written as $\Psi_{F}\left(x_{1}, \cdots, x_{N}\right)=$ $A\left(x_{1}, \cdots, x_{N}\right) \prod_{j=1}^{N} \phi\left(x_{j}\right)$, and the corresponding reduced one-body density matrix (OBDM) may be expressed as $\rho_{1}\left(x, x^{\prime}\right)=N \phi(x) \phi^{*}\left(x^{\prime}\right)\left[F\left(x, x^{\prime}\right)\right]^{N-1}$ where $F\left(x, x^{\prime}\right)=\int_{-\infty}^{\infty} \operatorname{sgn}\left(x-x^{\prime \prime}\right) \operatorname{sgn}\left(x^{\prime}-x^{\prime \prime}\right)\left|\phi\left(x^{\prime \prime}\right)\right|^{2} d x^{\prime \prime}[20]$. The single-particle momentum spectrum $n(k)$ for the Fermi gas may be obtained by Fourier transformation of the OBDM [14], and whereas the density $\rho(x)=$ $\rho_{1}(x, x)=N|\phi(x)|^{2}$ is the same for both the Fermi and mapped Bose systems, the momentum spectra of the two systems differ greatly [14].

$a_{1 D}<0$ : In this case $g_{1 D}^{F}>0$ and the mapped LL boson interactions are repulsive, $g_{1 D}^{B}>0$ [18]. The ground state solution of the GPE (3) with hard-wall boundary conditions is a dark solitary wave whose spatial form is an elliptic Jacobi snoidal function: Exhaustive details of the form of the solutions are given in Ref. [21]. In the limit of a single atom the GPE ground state degenerates into a half period of a sine function over the length $L$ of the trap. In contrast, for a large number of atoms $N>>1$ the solution tends to the value $\sqrt{n}$ within the center of the trap, but approaches the classic hyperbolic tangent form of a dark soliton near the hard wall boundaries within a distance characterized by the healing length $\xi_{h}=\sqrt{\left|a_{1 D}\right| /(2 n)}<<L$ which follows from the relation $\hbar^{2} /\left(2 m \xi_{h}^{2}\right)=g_{1 D}^{B} n[21]$.

Figure 2 shows the calculated scaled density profile $\rho(x) L$ for $N=14$ fermions and $\gamma_{B}=0.1$ for the case $a_{1 D}<0$ (dashed line) and also the ideal FTG gas (solid line) and we see that the density profile is broadened by the finite fermion interactions. For the ideal FTG gas the competing effects of the infinitely strong attractive 1D interactions and repulsion due to Fermi degeneracy pressure exactly cancel and that is why the ideal

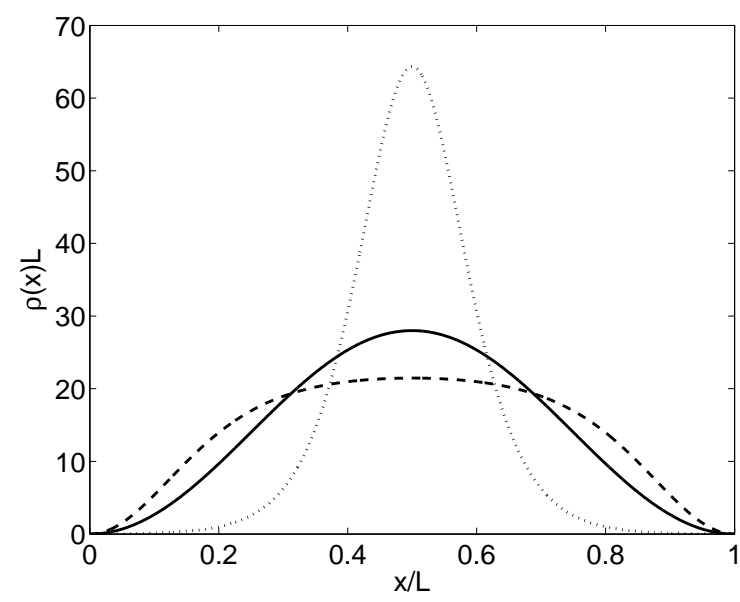

FIG. 2: Scaled density profile $\rho(x) L$ versus scaled coordinate $x / L$ for the ideal FTG (solid line), finite $a_{1 D}<0$ (dashed line), and finite $a_{1 D}>0$ (dotted line). The results are for $N=14$ and $\left|\gamma_{B}\right|=0.1$.

FTG maps to the ideal Bose gas with no residual interactions. In contrast, for the case considered here with finite but large $a_{1 D}<0$, the Fermi degeneracy pressure dominates over the attractive interactions and that is why the FTG is mapped to a LL model with repulsive interactions that broaden the density profile. This broadening of the density profile in the presence of decreasing attractive induced 1D fermion interactions was first found by Hao, Zhang, and Chen 14] using exact solutions for the fermion system based on the Bethe ansatz. Here we have elucidated the underlying physics by exposing the relation to the mapped LL model with repulsive interactions and its dark solitary wave solution.

Figure 3 shows the scaled momentum spectrum $2 \pi n(k) / L$ versus scaled momentum $k L / 2 \pi$ for $\left|\gamma_{B}\right|=0.1$, $N=14$ fermions, and $a_{1 D}<0$ (solid line). Bender, Erker, and Granger [20] have previously shown that for the ideal FTG the high momentum tails of the momentum spectrum are intimately related to the decay of the OBDM $\rho_{1}\left(x, x^{\prime}\right)$ as one moves off-diagonal $x \neq x^{\prime}$. In the thermodynamic limit they found that the decay of the correlations is exponential with decay constant $2 n$, and in our notation the scaled momentum spectrum becomes

$$
\frac{2 \pi n(k)}{L}=\frac{4 n^{2}}{\left(4 n^{2}+k^{2}\right)} .
$$

Thus, the larger the density $n$ is the more rapidly the correlations decay, and the larger the width $\Delta k=4 n$ of the momentum spectrum is. The dotted line in Fig. 3 shows the momentum spectrum using Eq. (44) and yields reasonable agreement with the high momentum tails in the numerical solution (solid line) even though we have a finite trap and are not in the thermodynamic limit.

$a_{1 D}>0$ : In this case $g_{1 D}^{F}<0$ and the mapped LL bo- 


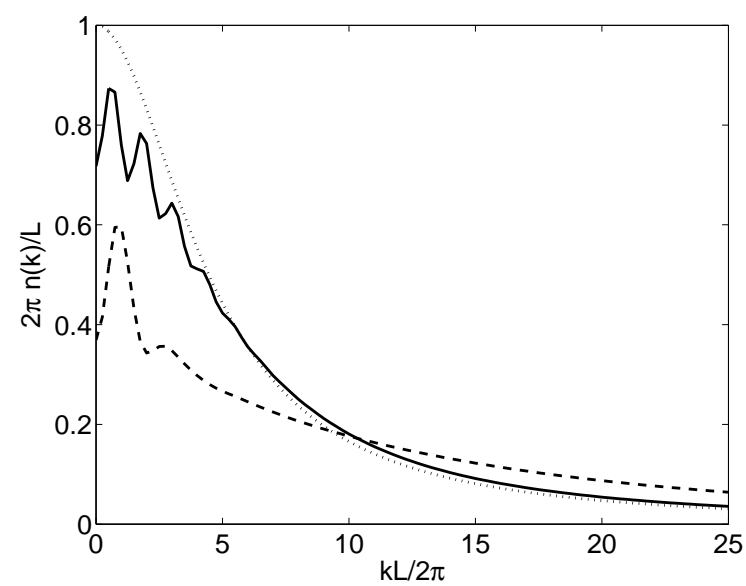

FIG. 3: Scaled momentum spectrum $2 \pi n(k) / L$ versus scaled momentum $k L / 2 \pi$ for $\left|\gamma_{B}\right|=0.1, N=14$ fermions, $a_{1 D}<0$ (solid line), $a_{1 D}>0$ (dashed line), and using Eq. (4) (dotted line).

son interactions are attractive, $g_{1 D}^{B}<0[18$. The ground state solution of the GPE (3) with hard-wall boundary conditions is a bright solitary wave whose spatial form is an elliptic Jacobi cnoidal function: Details of the form of the solutions are given in Ref. [22]. In the limit of a single atom the ground state solution degenerates into a half period of a sine function over the length $L$ of the trap, whereas for a large number of atoms $N>>1$ the solution becomes a localized peak at the center of the trap and approaches the classic hyperbolic secant form of a bright soliton with a characteristic width $w<L$ determined by $\hbar^{2} /\left(2 m w^{2}\right)=\left|g_{1 D}^{B}\right| N / w$ [22]. Thus, for a large number of atoms the bright soliton width becomes independent of the trap length $L, w=a_{1 D} /(4 N)$.

Figure 2 shows the calculated scaled density profile $\rho(x) L$ for $N=14$ fermions and $\left|\gamma_{B}\right|=0.1$ for finite $a_{1 D}>0$ (dotted line) and also the ideal FTG gas (solid line) and we see that the density profile is narrowed. This can be intuited using the fact that the mapped LL model has attractive interactions so that the density profile is narrowed compared to the free Bose gas, which maps to the ideal FTG.

Figure 3 shows the scaled momentum spectrum $2 \pi n(k) / L$ versus momentum $k L / 2 \pi$ for $\left|\gamma_{B}\right|=0.1, N=$ 14 fermions, and $a_{1 D}>0$ (dashed line), and by comparison with the case $a_{1 D}<0$ (solid line) the momentum spectrum is broader. This may be understood as follows: Inspection of the plots (not shown) of the OBDM $\rho_{1}\left(x, x^{\prime}\right)$ for $a_{1 D}>0$ shows that it decays significantly more rapidly as one moves off-diagonal $x \neq x^{\prime}$ in comparison to the case $a_{1 D}<0$, which implies that the momentum spectrum will be broader [20], see the discussion surrounding Eq. (4). This is also consistent with the fact that the peak density of the bright solitary wave is higher than that for the dark solitary wave, see Fig. 2, and the momentum spectrum width $\Delta k$ increases with density.
In summary, we have shown that both bright and dark solitary waves can arise in a one-dimensional spinpolarized gas of fermionic atoms with attractive 3D pwave interactions in a hard-wall trap, by virtue of the fact that the fermion system can be mapped to a system of interacting bosons with induced 1D interactions that can be either attractive or repulsive, exposing the underlying solitary waves.

* Electronic address: girardeau@optics.arizona.edu

$\dagger$ Electronic address: ewan.wright@optics.arizona.edu

[1] M. Olshanii, Phys. Rev. Lett. 81, 938 (1998).

[2] B.E. Granger and D. Blume, Phys. Rev. Lett. 92, 133202 (2004).

[3] J.L. Roberts et al., Phys. Rev. Lett. 86, 4211 (2001).

[4] B. Paredes, et al., Nature 429, 277 (2004); T. Kinoshita, T.R. Wenger, and D.S. Weiss, Science 305, 1125 (2004).

[5] T. Kinoshita, T.R. Wenger, and D.S. Weiss, Phys. Rev. Lett. 95, 190406 (2005).

[6] T. Kinoshita, T.R. Wenger, and D.S. Weiss, Nature 440, 900 (2006).

[7] M. Girardeau, J. Math. Phys. 1, 516 (1960); M.D. Girardeau, Phys. Rev. 139, B500 (1965), Secs. 2, 3, and 6.

[8] M.D. Girardeau and M. Olshanii, cond-mat/0309396.

[9] M.D. Girardeau, Hieu Nguyen, and M. Olshanii, Optics Communications 243, 3 (2004).

[10] C. Ticknor, C.A. Regal, D.S. Jin, and J.L. Bohn, Phys. Rev. A 69, 042712 (2004).

[11] T. Cheon and T. Shigehara, Phys. Lett. A 243, 111 (1998) and Phys. Rev. Lett. 82, 2536 (1999).

[12] M.D. Girardeau and E.M. Wright, arXiv:0711.0459v2 [cond-mat.other] (2007).

[13] E.H. Lieb and W. Liniger, Phys. Rev. 130, 1605 (1963).

[14] Yajiang Hao, Yunbo Zhang, and Shu Chen, arXiv:0708.3548 [cond-mat.other] (2007).

[15] M.D. Girardeau and E.M. Wright, Phys. Rev. Lett. 84, 5691 (2000).

[16] T. Karpiuk et. al., Phys. Rev. A 66, 023612 (2002).

[17] E. Witkowska and M. Brewczyk, Phys. Rev. A 72, 023606 (2005).

[18] $g_{1 D}^{F}$ is sometimes defined with the opposite sign, in which case one would have instead $g_{1 D}^{B} g_{1 D}^{F}=-\frac{4 \hbar^{4}}{m^{2}}$. The sign of $g_{1 D}^{F}$ has no intrinsic physical significance, whereas that of $a_{1 D}$ does via Eq. (2) and Fig 1] The total energy (kinetic plus potential) and $a_{1 D}$ are invariant under the FB mapping, negative $a_{1 D}$ implies positive total energy and an unbound ground state, and positive $a_{1 D}$ implies negative total energy and a bound ground state.

[19] See, for example, E. M. Lifshitz and L. P. Pitaevskii, Statistical Physics, Part 2 (Pergamon Press, Oxford, 1989)pp. 85-118.

[20] Scott A. Bender, Kevin D. Erker, and Brian E. Granger, Phys. Rev. Lett. 95, 230404 (2005).

[21] L. D. Carr, C. W. Clark, and W. P. Reinhardt, Phys. Rev. A 62, 063610 (2000).

[22] L. D. Carr, C. W. Clark, and W. P. Reinhardt, Phys. Rev. A 62, 063611 (2000). 